INPLASY

PROTOCOL

To cite: Niu et al. A metaanalysis of the efficacy of selenium in the treatment of Graves' disease. Inplasy protocol 202140144. doi: 10.37766/inplasy2021.4.0144

Received: 28 April 2021

Published: 29 April 2021

Corresponding author:

Na Niu

beimengxueying@163.com

Author Affiliation:

Tianjin Xiqing Hospital

Support: None.

Review Stage at time of this submission: Preliminary searches.

Conflicts of interest:

None declared.

\section{A meta-analysis of the efficacy of selenium in the treatment of Graves' disease}

\author{
Niu, N1; Li, SY2; Zheng, J. ${ }^{3}$
}

Review question / Objective: The review of this system aims to explore the clinical effect of selenium in the treatment of Graves' disease.

Condition being studied: Graves disease is a common autoimmune thyroid disease, which seriously affects the quality of life of patients. At present, the main treatment methods include drugs and surgery. The main drugs are antithyroid drugs. There is currently limited evidence that the combination of trace element selenium is beneficial for the treatment of the disease.

Information sources: The medical databases searched in this study include (searching related journals, conference records, dissertations): CNKI, Wanfang database, Weipu database, CBM database, PubMed, Cochrane Library, Embase, Web of Science, MEDLINE, etc.

INPLASY registration number: This protocol was registered with the International Platform of Registered Systematic Review and Meta-Analysis Protocols (INPLASY) on 29 April 2021 and was last updated on 29 April 2021 (registration number INPLASY202140144).

\section{INTRODUCTION}

Review question / Objective: The review of this system aims to explore the clinical effect of selenium in the treatment of Graves' disease.
Condition being studied: Graves disease is a common autoimmune thyroid disease, which seriously affects the quality of life of patients. At present, the main treatment methods include drugs and surgery. The main drugs are antithyroid drugs. There is 
currently limited evidence that the combination of trace element selenium is beneficial for the treatment of the disease.

\section{METHODS}

Participant or population: Participants must be diagnosed with a standard Graves disease. There are no limits to research subjects' age, ender, race.

Intervention: The experimental group was treated with selenium combined with antithyroid drugs.

Comparator: The control group was treated with antithyroid drugs.

Study designs to be included: Only Randomized Controlled Clinical Trials (RCT)-selenium treatment of Graves disease related content will be included in this systematic review. Trials published in the form of papers will also be selected as qualified studies.

Eligibility criteria: Interventions include any clinical medicine containing selenium.

Information sources: The medical databases searched in this study include (searching related journals, conference records, dissertations): CNKI, Wanfang database, Weipu database, CBM database, PubMed, Cochrane Library, Embase, Web of Science, MEDLINE, etc.

Main outcome(s): Mainly compare the levels of TPOAB, TGAB, and TRAB in the treatment group and the observation group after treatment, and evaluate the effectiveness of the treatment.

Quality assessment / Risk of bias analysis: We will use the Cochrane Collaboration's tool which is recommended by the Cochrane Reviewer's Handbook to assess risk of bias for quality assessment of the included studies. The studies will be graded based on: (i) random sequence generation; (ii) allocation concealment; (iii)blinding; (iv) incomplete outcome data; (v) selective outcome reporting; (vi) other sources of bias.
Strategy of data synthesis: The efficacy of any form of selenium combined with antithyroid drugs and the same anti-thyroid drugs in the treatment of Graves' disease will be compared. The included research data may be divided into two cases, depending on whether the data is suitable for meta-analysis. If meta-analysis is not possible due to heterogeneity, we will describe it qualitatively. If the data is suitable for meta-analysis, we will use RevMan 5.3 (Review Manager) software for meta-analysis. For dichotomy data, we express the result as an OR value with a $95 \%$ confidence interval (CI). For continuous data, the mean difference (MD). If the outcome variables are measured on different scales, standard mean difference (SMD) analysis with $95 \% \mathrm{Cl}$ will be performed. For whether the data exists in the meta-analysis, the heterogeneity will be tested by the standard $I^{2}$ test. If there is no statistical heterogeneity between the results, a fixed effects model is used for meta-analysis. If there are statistical differences, further analysis of heterogeneity should be done. If there is significant clinical heterogeneity, a subgroup or sensitivity analysis can be performed, or only a descriptive analysis can be performed. Sensitivity analysis detects the stability of the results of the study, and uses a funnel chart to observe publication bias.

Subgroup analysis: If there is significant heterogeneity in the included trials, we will conduct a subgroup analysis based on the specific circumstances (such as the length of the disease course, the severity of the disease, etc.).

Sensitivity analysis: When there are sufficient studies, we will carry out sensitivity analysis to test the robustness of studies according to the quality of method, the sample size and the selection of missing data. And the fluctuation of results will be observed.

Language: Without any language or publication status restrictions.

Country(ies) involved: China. 
Keywords: Selenium, Methimazole, Graves disease, RCT, Meta analysis.

Contributions of each author:

Author 1 - Na Niu.

Author 2 - Shuying Li.

Author 3 - Jie Zheng. 